\title{
Associação entre a classificação da deambulação funcional com a velocidade da marcha após acidente vascular cerebral
}

\section{Association between the classification of functional ambulation classification and gait speed after stroke}

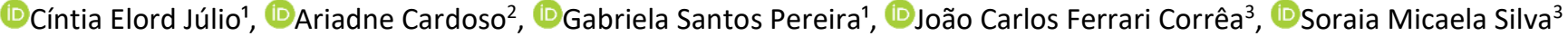

1 Doutoranda em Ciências da Reabilitação, Universidade Nove de Julho - UNINOVE

2 Fisioterapeuta, Universidade Nove de Julho UNINOVE

3 Docente, Programa de Pós-Graduação em Ciências da Reabilitação, Universidade Nove de Julho - UNINOVE

Correspondência

Cíntia Elord Júlio

E-mail: cintia elord@hotmail.com

Submetido: 13 Julho 2020

Aceito: 30 Setembro 2020

\section{Como citar}

Júlio CE, Cardoso A, Pereira GS, Corrêa JCF, Silva SM. Associação entre a classificação da deambulação funcional com a velocidade da marcha após acidente vascular cerebral. Acta Fisiatr. 2020;27(2):95-99.

DOI: 10.11606/issn.2317-0190.v27i2a172364

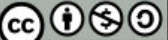

BY NC SA

Este trabalho está licenciado com uma licença

Creative Commons - Atribuição 4.0 Internacional

\section{RESUMO}

A escolha do instrumento adequado é decisiva para o sucesso da avaliação funcional e reabilitação. Diante disto, é necessário que o profissional tenha amplo conhecimento dos instrumentos disponíveis para que possa optar pela avaliação mais eficaz, menos onerosa e mais rápida. Objetivo: Analisar se a classificação da deambulação obtida pela Functional Ambulation Classification (FAC) se associa com o desempenho obtido no Teste de Caminhada de 10 metros (TC10M). Métodos: Estudo transversal no qual foram avaliados 61 indivíduos acometidos pelo Acidente Vascular Cerebral (AVC). Utilizou-se o TC10M e a FAC. Para verificar a normalidade dos dados foi utilizado teste Kolmogorov-Smirnov. Foi utilizado o ANOVA de uma via para verificar se houve diferença dos deambuladores dependentes e independentes conforme a FAC com a velocidade da marcha por meio do TC10M. Foi realizado um teste post hoc e o método de Scheffe, considerando $p \leq 0,05$. Para analisar a associação da categorização da deambulação com a velocidade da marcha, foi o utilizado o teste do qui-quadrado de independência. Resultados: Houve diferença significante de cada nível da FAC em relação a velocidade da marcha. Houve associação entre as classificações obtidas pela FAC e pelo TC10m [x2(9) $=145,335 ; p=0,001]$; sendo que em $48 \%$ as variáveis estão corretamente associadas. Conclusão: Os achados indicaram que os níveis da FAC apresentaram associação com a velocidade da marcha. Este achada amplia os conhecimentos sobre medidas de avaliação da marcha e auxilia a prática clínica, considerando que facilita a escolha do melhor instrumento para avaliar a marcha após AVC.

Palavras-chave: Acidente Vascular Cerebral, Marcha, Caminhada, Avaliação de Processos e Resultados

\section{ABSTRACT}

The choice of the appropriate instrument is decisive for the success of functional assessment and rehabilitation. In view of this, it is necessary that the professional has extensive knowledge of the instruments available so that he can choose the most effective, least costly and quickest assessment. Objective: To analyze whether the classification of ambulation obtained by the Functional Ambulation Classification (FAC) is associated with the performance obtained in the 10-meter walk test (TC10m). Methods: Cross-sectional study in which 61 individuals affected by stroke were evaluated. TC10m and FAC were used. To verify the normality of the data, a Kolmogorov-Smirnov test was used. One-way ANOVA was used to check whether there was a difference between dependent and independent walkers according to FAC with gait speed using the TC10m. $A$ post hoc test and the Scheffe method were performed, considering $p \leq 0.05$. To analyze the association between the categorization of walking and gait speed, the chi-square test of independence was used. Results: There was a significant difference at each FAC level in relation to gait speed. There was an association between the classifications obtained by the FAC and by the TC10m $[\times 2(9)=145.335 ; \mathrm{p}=0.001]$; and in $48 \%$ the variables are correctly associated. Conclusion: The findings indicated that the FAC levels were associated with walking speed. This finding expands the knowledge about gait assessment measures and helps clinical practice, considering that it facilitates the choice of the best instrument to assess gait after stroke.

Keywords: Stroke, Gait, Walking, Outcome and Process Assessment, Health Care 


\section{INTRODUÇÃO}

O Acidente Vascular Cerebral (AVC) é a segunda principal causa de morte e a terceira principal causa de incapacidade funcional no mundo, ${ }^{1,2}$ sendo definido como uma disfunção neurológica aguda de origem vascular, seguida de acometimento súbito com sinais e sintomas relacionados aos comprometimentos de áreas focais ou globais no cérebro que pode ocorrer devido a uma obstrução de uma artéria (AVC isquêmico) ou por ruptura de um vaso (AVC hemorrágico). ${ }^{3}$

Os déficits motores decorrentes do AVC caracterizam-se por hemiplegia ou hemiparesia, tipicamente no lado do corpo oposto ao local da lesão. A deficiência dos movimentos pode levar a limitações funcionais e incapacidades, como a perda de mobilidade no tronco e nas extremidades, padrões atípicos de movimento, estratégias compensatórias e ações involuntárias do hemicorpo afetado levando a perda da independência na vida diária. ${ }^{3}$

Em decorrência dos déficits motores, a marcha é uma das atividades mais afetadas, acarretando redução da velocidade e maior risco de quedas. Logo, intervenções voltadas para a melhora da marcha, assim como aumento da velocidade, são amplamente estudadas. ${ }^{4,5}$ Assim, faz-se necessária uma avaliação detalhada para que práticas clínicas sejam melhores desenvolvidas na reabilitação destes indivíduos.

Diversos instrumentos são utilizados para avaliar a marcha, alguns clínicos outros laboratoriais e os que apresentam maior frequência de utilização nos estudos são: velocidade da marcha, parâmetros espaço - temporais, Índice de Barthel, cinemática, a Functional Ambulation Classification (FAC), cinética, teste de caminhada de 6 minutos (TC6), teste da caminhada de 10 metros (TC10M), Timed Up and Go e eletromiografia. ${ }^{6}$

Dentre estas, destaca-se a FAC, que é uma escala de classificação de deambulação amplamente utilizada na prática clínica, criada em 1984 por Holden et al. A FAC avalia a capacidade de andar utilizando 6 níveis de deambulação baseada na dificuldade e na necessidade de suporte requerido durante a marcha. ${ }^{7-9}$ É um instrumento de avaliação rápido, aplicável a rotina clínica, de fácil interpretação, baixo custo e eficaz. ${ }^{7-9}$ Apresenta adequada confiabilidade, validade concorrente e reprodutibilidade para população brasileira, ${ }^{10}$ além de ser parâmetro para acompanhar a evolução da marcha na reabilitação de forma categórica. ${ }^{8}$

Foi testada em pacientes com AVC e esclerose múltipla, $7,9,10$ entretanto, para classificação do nível de deambulação do paciente é necessário a experiência clínica do avaliador, uma vez, que pode haver uma análise subjetiva, além de necessitar de um espaço físico amplo, o que pode dificultar a avaliação funcional da marcha. ${ }^{10}$

Portanto, faz-se necessário outras opções de avaliação da marcha, que melhor se adeque à necessidade do profissional. A escolha do instrumento adequado é decisiva para o sucesso de uma boa avaliação funcional e para avaliar a eficácia do tratamento. ${ }^{11}$

Diante disto, é necessário que o profissional tenha amplo conhecimento dos instrumentos disponíveis para que possa optar pela avaliação mais eficaz, menos onerosa e mais rápida possível. A hipótese é que a FAC tenha associação com a velocidade da marcha, e que assim avalie construtos similares e o profissional possa optar pelo instrumento que melhor atender suas necessidades.

\section{OBJETIVO}

O objetivo deste estudo foi analisar se a classificação da deambulação obtida pela FAC se associa com o desempenho obtido no TC10M.

\section{MÉTODOS}

Estudo transversal no qual foram avaliados 61 indivíduos acometidos pelo AVC recrutados da clínica de fisioterapia da Universidade Nove de Julho.

Para serem incluídos no estudo os indivíduos deveriam apresentar diagnóstico de AVC crônico (> 6 meses) ${ }^{12}$ e apresentar cognitivo preservado, avaliado pelo mini exame do estado mental (MEEM) sendo o ponto de corte acima ou igual a 13 pontos para indivíduos analfabetos, 18 pontos pra baixa/média escolaridade e 26 pontos pra alta escolaridade. ${ }^{13}$

Indivíduos que apresentaram afasia, patologias ortopédicas ou outras neurológicas além do AVC que alterassem a habilidade de locomoção e aqueles que não apresentavam nenhum comprometimento motor que alterasse a habilidade de locomoção foram excluídos.

Este estudo foi aprovado pelo Comitê de Ética em Pesquisa da Universidade Nove de Julho (UNINOVE) de São Paulo, SP, Brasil (CAEE 79057817.4.0000.5511). Todos os envolvidos e os responsáveis legais dos voluntários assinaram o termo de consentimento livre e esclarecido informado após as explicações e concordaram em participar do estudo.

\section{Teste de caminhada de 10 metros - TC10M}

Para verificar a velocidade da marcha, utilizou - se o TC10M que é um teste que mostrou excelente confiabilidade para pacientes com AVC. ${ }^{14}$

Foi escolhido o 10MWT pois a FAC se correlaciona significantemente com medidas de capacidade da marcha, como a velocidade, que para cada nível da FAC apresentou diferença significativa. Além disso, os testes de marcha são utilizados com muita frequência na reabilitação, em pesquisas e são indicadores de progresso na performance da marcha.

Neste teste o paciente teve que caminhar em solo regular a uma distância de 10 metros em sua velocidade usual, podendo fazer uso de dispositivo auxiliar de marcha. $O$ teste foi realizado 3 vezes e o melhor desempenho foi considerado. ${ }^{14}$

\section{Classificação de deambulação funcional - FAC}

Na FAC a marcha é categorizada em 6 níveis de deambulação:

Nível "1" (não funcional) indica que o paciente não é capaz de deambular ou requer assistência física ou supervisão de mais de uma pessoa, ou só deambula em barras paralelas.

Nível "2" (dependente nível 2) indica um paciente que requer contato manual de uma pessoa sendo este contato contínuo para suportar o peso corporal, bem como para manter o equilíbrio ou para auxiliar na coordenação. 
Nível " 3 " (dependente nível 1) indica que o paciente requer o contato manual leve, intermitente ou contínuo de uma pessoa para auxiliar no equilíbrio ou coordenação em solo regular.

Nível "4" (dependente, supervisão) indica um paciente que pode deambular em solo regular, sem contato manual de outra pessoa, mas por segurança, requer uma pessoa ao lado, por causa do comprometimento na capacidade de decisão, status cardíaco questionável, ou da necessidade de verbalização para completar a tarefa.

Nível " 5 " (independente apenas em superfícies niveladas) indica que o paciente deambula de forma independente em superfície plana, mas requer supervisão ou contato manual para superfícies irregulares.

Nível " 6 " (independente em superfícies niveladas e desniveladas) indica que paciente deambula em superfícies regulares e irregulares de forma independente. ${ }^{11}$

\section{Análise estatística}

Para verificar a normalidade dos dados foi utilizado teste Kolmogorov - Smirnov. Para a caracterização da amostra e distribuição dos dados foi utilizada a estatística descritiva. As variáveis paramétricas foram representadas por média e desvio padrão, as não paramétricas em mediana e intervalo interquartílico e as variáveis categóricas representadas em frequência.

Para análise da associação da categorização da deambulação com a FAC e com a velocidade da marcha, classificou-se a deambulação em domiciliar quando a velocidade durante o TC10M foi $<0,4 \mathrm{~m} / \mathrm{s}$, deambulação comunitária limitada entre 0,4 e $0,8 \mathrm{~m} / \mathrm{s}$ e deambulação comunitária $>0,8 \mathrm{~m} / \mathrm{s}^{15}$ e estas classificações foram associadas com os níveis da FAC caracterizando os indivíduos como: deambulador não funcional (Nível 1 da FAC), deambulador dependente (Nível 2 e 3 da FAC), deambulador dependente com supervisão (Nível 4 da FAC) e deambulador Independente (Nível 5 e 6 da FAC). Os dados foram log transformados e foi utilizado o ANOVA de uma via para verificar se houve diferença dos deambuladores dependentes e independentes conforme a FAC com a velocidade da marcha por meio do TC10M. Foi realizado também um teste post hoc e o método de Scheffe. Sendo considerado nível de significância de $p \leq 0,05 .{ }^{16}$

Para analisar a associação da categorização da deambulação feita pela FAC e pela velocidade da marcha foi o utilizado o teste do qui-quadrado, considerando um $p \leq 0,05$, seguido do teste de força $V$ de Cramer por ser o mais usado para testar os dados, caso o resultado obtido do qui-quadrado seja significante. ${ }^{17}$ Para análise estatística utilizou-se IBM Corp.
Released 2013. IBM SPSS Statistics for Windows, Version 22.0. Armonk, NY: IBM Corp.

\section{RESULTADOS}

Foram triados 71 indivíduos com hemiparesia crônica, destes quatro foram excluídos devido à déficit cognitivo (pontuação menor que 11 pontos no MEEM) e seis por apresentaram outras patologias ortopédicas que alteravam a habilidade de locomoção.

Sendo assim, a amostra final foi composta por 61 indivíduos que foram avaliados nos ambulatórios de fisioterapia da Universidade Nove de Julho cujas características clínicodemográficas estão apresentadas na Tabela 1.

Tabela 1. Características clínico-demográficas dos participantes do estudo

\begin{tabular}{lc}
\hline \multicolumn{1}{c}{ Variável } & $\mathbf{n}=\mathbf{6 1}$ \\
\hline Gênero & $37(60,7 \%)$ \\
Homens & $24(39,3 \%)$ \\
Mulheres & $57,30 \pm 12,70$ \\
Idade (anos) & $24(12-54)$ \\
Tempo após AVC (meses) & \\
Hemicorpo acometido & $30(49,2 \%)$ \\
Direito & $30(49,2 \%)$ \\
Esquerdo & $1(1,6 \%)$ \\
Ambos & \\
Tipo de AVC & $46(75,4 \%)$ \\
Isquêmico & $15(24,6 \%)$ \\
Hemorrágico & $12(4,5-12)$ \\
Escolaridade (anos) & $25(22-27,5)$ \\
Mini-Mental(pontos)
\end{tabular}

A variável idade foi expressa em média e desvio padrão. Idade, escolaridade e mini mental foram expressas em mediana e intervalo interquartílico; as demais variáveis em freqüência

Observou-se diferença significante $(F=9,81 \mathrm{p}<0,001) \mathrm{da}$ velocidade da marcha para cada nível de deambulação conforme a FAC. Nota-se ainda, que o nível 1 obteve menor velocidade $(0,02 \mathrm{~m} / \mathrm{s})$ em detrimento aos níveis $5(0,53 \mathrm{~m} / \mathrm{s})$ e 6 $(0,78 \mathrm{~m} / \mathrm{s})$. O teste pos hoc evidenciou que todos os níveis da FAC apresentaram diferença significante da velocidade da marcha, inclusive entre os níveis 5 e 6 (Diferença das médias $(D M)=-0,16 ; p=0,04)$.

$\mathrm{Na}$ associação da categorização da deambulação feita pela FAC e pela velocidade da marcha, foi observado por meio do teste do qui-quadrado de independência que há associação entre as classificações obtidas pela FAC e pela velocidade da marcha $(\mathrm{TC} 10 \mathrm{~m})[\times 2(9)=145,335 ; \mathrm{p}=0,001]$; sendo que em $48 \%$ as variáveis estão corretamente associadas (V de Cramer) (Tabela 2).

Tabela 2. Associação entre a classificação da deambulação obtida com a FAC e a categorização de acordo com a velocidade da marcha

\begin{tabular}{|c|c|c|c|c|c|}
\hline \multirow[b]{2}{*}{$\begin{array}{l}\text { Categorização de acordo com a } \\
\text { velocidade da marcha }\end{array}$} & \multicolumn{5}{|c|}{ Categorização da FAC } \\
\hline & $\begin{array}{l}\text { Deambulador não } \\
\text { funcional (FAC 1) }\end{array}$ & $\begin{array}{c}\text { Deambulador } \\
\text { dependente (FAC } 2 \text { e 3) }\end{array}$ & $\begin{array}{c}\text { Deambulador dependente } \\
\text { com supervisão (FAC 4) }\end{array}$ & $\begin{array}{c}\text { Deambulador } \\
\text { Independente (FAC } 5 \text { e } 6 \text { ) }\end{array}$ & (n) \\
\hline$<0,4 \mathrm{~m} / \mathrm{s}$ Deambulador domiciliar & 0 & 1 & 1 & 8 & 10 \\
\hline 0,4 a $0,8 \mathrm{~m} / \mathrm{s}$ Deambulador comunitário limitado & 0 & 0 & 0 & 21 & 21 \\
\hline$>0,8 \mathrm{~m} / \mathrm{s}$ Deambulador comunitário & 0 & 0 & 0 & 14 & 14 \\
\hline $0 \mathrm{~m} / \mathrm{s}$ Não deambulador & 9 & 4 & 2 & 1 & 16 \\
\hline Total & 9 & 4 & 2 & 44 & 61 \\
\hline
\end{tabular}




\section{DISCUSSÃO}

De acordo com os resultado obtidos, pode-se inferir que a FAC é capaz de discriminar indivíduos com diferentes tipos de deambulação, tendo em vista que há diferença significante na velocidade da marcha de cada nível de deambulação da FAC. Observou-se que os indivíduos que não deambulavam ou aqueles que não conseguiam deambular a uma distância mínima de 10 metros foram classificados como não deambuladores e a maioria destes indivíduos estavam nos níveis 1,2 e 3 da FAC.

Os indivíduos que foram classificados nas categorias de deambulação comunitária limitada e deambulação comunitária, a maioria estavam nos níveis 5 e 6 da FAC. Isso demonstra que um melhor desempenho no TC10m é condizente com a classificação de indivíduos em níveis mais altos da FAC como 5 e 6.

Holden MK et al. ${ }^{7,9}$ e Júlio CE et al. ${ }^{10}$ também identificaram diferença significante ao contrastar a velocidade da marcha de indivíduos com diferentes classificações da FAC. Identificou-se ainda, que há associação significante entre cada nível de classificação da deambulação da FAC com as classificações obtidas pela velocidade da marcha, sendo que em $48 \%$ das vezes os indivíduos são classificados corretamente, considerando o desempenho no TC10m. Isso significa que ambos os instrumentos avaliam construtos semelhantes $e$ portanto, o profissional pode optar pelo instrumento que melhor se adequar ao seu objetivo.

Embora a FAC tenha sido criada inicialmente para indivíduos na fase aguda da lesão, ainda em ambientes hospitalares, ${ }^{7,9}$ Mehrholz J et al. ${ }^{8}$ sugeriram que a FAC pode ser sensível e específica para a previsão da marcha na comunidade.

No estudo de Julio et al. ${ }^{10}$ observou-se que a escala pode ser utilizada em ambiente clínico e o presente estudo conseguiu reforçar essa utilização devido as características da amostra, no qual foram selecionados indivíduos com AVC na fase crônica. ${ }^{10}$ Porém, este fator também gerou limitações no estudo, uma vez que a amostra não se distribuiu de forma homogênea em todos os níveis da FAC, se concentrando mais nos níveis mais independentes ( 5 e 6$)$, e isso pode ter influenciado nos resultados.

Contudo, cabe destacar que apesar desta associação significante, identificou-se algumas inconsistências dos dados quando comparado a classificação pela velocidade da marcha. Observou-se que indivíduos classificados como deambuladores independentes pela FAC foram classificados como deambuladores domiciliar $(n=8)$, deambuladores comunitários limitado $(n=21)$ e deambuladores comunitários $(n=14)$.

Essa variação de classificação pode ter acontecido devido as estratégias compensatórias desenvolvidas pelos indivíduos com AVC crônico, que mesmo deambulando com menor velocidade no TC10m podem ser caracterizados como independentes pela FAC.

Observou-se ainda, que indivíduos que apresentaram velocidade de $0 \mathrm{~m} / \mathrm{s}$ (não deambulador) foram classificados nos níveis 2,4 e 5 da FAC (níveis que indicam deambulação), isso deve-se ao fato de que alguns indivíduos não conseguiram deambular por $10 \mathrm{~m}$ no TC10m, porém, conseguiram realizar o percurso de 3 metros exigidos na FAC.
Estes achados contrastam com os resultados demonstrados no estudos de Mehrholz J et al. ${ }^{8}$ que pode ser explicado pelo fato dos autores terem avaliado uma amostra de pacientes acometidos pelo AVC em fases aguda e subaguda. Outra limitação foi a utilização de somente uma variável como a velocidade da marcha (TC10m) ter sido associada aos níveis da FAC, sendo que outras variáveis poderiam ter sido investigadas para justificar ainda mais a utilização da FAC.

O risco de quedas é um fator importante a ser avaliado, uma vez que estudos demonstram a relação da diminuição da velocidade da marcha com um aumento do risco de quedas em idosos, assim como uma piora do equilíbrio dinâmico e uma maior predisposição a fragilidade. ${ }^{18,19}$ Portanto, talvez a utilização do Timed Up and Go Test ${ }^{20}$ que avalia estas variáveis poderia ter sido outro instrumento a ser utilizado neste estudo para verificar a associação com os níveis da FAC.

\section{CONCLUSÃO}

Conclui-se que apesar das limitações do estudo, os níveis da FAC associação é capaz de discriminar diferentes tipos de deambulação comunitária, além de apresentar associação com o desempenho do TC10m. Este achado amplia os conhecimentos sobre medidas de avaliação da marcha e auxilia a prática clínica dos profissionais da reabilitação, considerando que facilita a escolha do melhor instrumento para avaliar a marcha após AVC.

\section{Agradecimentos}

Os autores agradecem à Agência de Fomento Brasileira de Coordenação e Aperfeiçoamento Pessoal de Nível superior (CAPES [Coordination for the Advanced of Higher Education Personnel]) e a Universidade Nove de Julho (UNINOVE).

\section{REFERÊNCIAS}

1. Johnson W, Onuma O, Owolabi M, Sachdev S. Stroke: a global response is needed. Bull World Health Organ. 2016;94(9):634-634A.

https://doi.org/10.2471/BLT.16.181636

2. Murray $C J$, Vos $T$, Lozano $R$, Naghavi $M$, Flaxman $A D$, Michaud C, et al. Disability-adjusted life years (DALYs) for 291 diseases and injuries in 21 regions, 1990-2010: a systematic analysis for the Global Burden of Disease Study 2010. Lancet. 2012;380(9859):2197-223. Doi: https://doi.org/10.1016/S0140-6736(12)61689-4

3. Asplund K, Sukhova M, Wester P, Stegmayr B; Riksstroke Collaboration. Diagnostic procedures, treatments, and outcomes in stroke patients admitted to different types of hospitals. Stroke. 2015;46(3):806-12. Doi: https://doi.org/10.1161/STROKEAHA.114.007212

4. Ray NT, Knarr BA, Higginson JS. Walking speed changes in response to novel user-driven treadmill control. J Biomech. 2018;78:143-9.

https://doi.org/10.1016/j.jbiomech.2018.07.035

Doi:

5. Kinoshita S, Abo M, Okamoto T, Tanaka N. Utility of the revised version of the ability for basic movement scale in predicting ambulation during rehabilitation in poststroke patients. J Stroke Cerebrovasc Dis. 2017;26(8):1663-1669. Doi: https://doi.org/10.1016/j.jstrokecerebrovasdis.2017.02.021 
6. Mudge S, Stott NS. Outcome measures to assess walking ability following stroke: a systematic review of the literature. Physiotherapy. 2007;93(3):189-200. Doi: https://doi.org/10.1016/j.physio.2006.12.010

7. Holden MK, Gill KM, Magliozzi MR. Gait assessment for neurologically impaired patients. Standards for outcome assessment. Phys Ther. 1986;66(10):1530-9. Doi: https://doi.org/10.1093/pti/66.10.1530

8. Mehrholz J, Wagner K, Rutte K, Meissner D, Pohl M. Predictive validity and responsiveness of the functional ambulation category in hemiparetic patients after stroke. Arch Phys Med Rehabil. 2007;88(10):1314-9. Doi: https://doi.org/10.1016/j.apmr.2007.06.764

9. Holden MK, Gill KM, Magliozzi MR, Nathan J, Piehl-Baker L. Clinical gait assessment in the neurologically impaired. Reliability and meaningfulness. Phys Ther. 1984;64(1):3540. Doi: https://doi.org/10.1093/ptj/64.1.35

10. Elord C, Corrêa FI, Pereira GS, Silva SM, Corrêa JC. Translation into Brazilian Portuguese, cross-cultural adaptation, reliability and validation of the Functional Ambulation Classification for the categorization of ambulation following a stroke in a clinical setting. Rev Neurol. 2020;70(10):365-71. Doi: https://doi.org/10.33588/rn.7010.2019400

11. Brandão TCP, Silva FP, Silva SM. Força de preensão manual prediz moderadamente a recuperação sensório-motora avaliada pela escala Fugl-Meyer. Fisioter Pesqui. 2018 25(4):404-09. Doi: https://doi.org/10.1590/18092950/17010125042018

12. Bernhardt J, Hayward KS, Kwakkel G, Ward NS, Wolf SL, Borschmann K, et al. Agreed definitions and a shared vision for new standards in stroke recovery research: the stroke recovery and rehabilitation roundtable taskforce. Int J Stroke. 2017;12(5):444-450. Doi: https://doi.org/10.1177/1747493017711816
13. Maheshwari SG, Iqbal M, Hashmi SFA, Devrajani B. Stroke patients; assessment of cognitive impairment in. Professional Med J. 2015;22(4):541-5.

14. Scrivener K, Schurr K, Sherrington C. Responsiveness of the ten-metre walk test, Step Test and Motor Assessment Scale in inpatient care after stroke. BMC Neurol. 2014;14:129. Doi: https://doi.org/10.1186/1471-2377-14-129

15. Khanittanuphong P, Tipchatyotin S. Correlation of the gait speed with the quality of life and the quality of life classified according to speed-based community ambulation in Thai stroke survivors. NeuroRehabilitation. 2017;41(1):135-41. Doi: https://doi.org/10.3233/NRE-171465

16. Krebs DE. Declare your ICC type. Phys Ther. 1986;66(9):1431. https://doi.org/10.1093/pti/66.9.1431

17. McHugh ML. The Chi-square test of independence. Biochem Med (Zagreb). 2013;23(2):143-9. Doi: https://doi.org/10.11613/BM.2013.018

18. Bedoya-Belmonte JJ, Rodríguez-González MDM, GonzálezSánchez M, Pitarch JMB, Galán-Mercant A, Cuesta-Vargas Al. Inter-rater and intra-rater reliability of the extended TUG test in elderly participants. BMC Geriatr. 2020;20(1):56. Doi: https://doi.org/10.1186/s12877-0201460-0

19. Barry E, Galvin R, Keogh C, Horgan F, Fahey T. Is the Timed Up and Go test a useful predictor of risk of falls in community dwelling older adults: a systematic review and meta-analysis. BMC Geriatr. 2014;14:14. Doi: https://doi.org/10.1186/1471-2318-14-14

20. Costa AGS, Oliveira ARS, Sousa VEC, Araújo TL, Cardoso MVLML, Silva VM. Instrumentos utilizados no brasil para avaliação da mobilidade física como fator preditor de quedas em adultos. Ciência, Cuidado e Saúde. 2012;10(2):401-7. Doi: https://doi.org/10.4025/cienccuidsaude.v10i2.12085 